Vorverstärker mit optischer Signalübertragung und optischer Leistungsversorgung für hochqualitative Elektrokardiogramme während Magnetresonanz-(MR)-Untersuchungen

Scheidegger, M B ; et al

DOI: https://doi.org/10.1515/bmte.1996.41.s1.286

Posted at the Zurich Open Repository and Archive, University of Zurich ZORA URL: https://doi.org/10.5167/uzh-154605

Journal Article

Published Version

Originally published at:

Scheidegger, M B; et al (1996). Vorverstärker mit optischer Signalübertragung und optischer Leistungsversorgung für hochqualitative Elektrokardiogramme während Magnetresonanz-(MR)-Untersuchungen. Biomedizinische Technik. Biomedical engineering, 41(s1):286-287.

DOI: https://doi.org/10.1515/bmte.1996.41.s1.286 


\title{
Vorverstärker mit optischer Signalübertragung und optischer Leistungsversorgung für hochqualitative Elektrokardiogramme während Magnetresonanz-(MR)-Untersuchungen
}

\author{
M.B. Scheidegger, M. Stuber, B. Stüssi, S. Fischer, P. Boesiger
}

Institut für Biomedizinische Technik und Medizinische Informatik, Universität und ETH Zürich, Schweiz

\begin{abstract}
THEMA
Für eine artefaktfreie Akquisition von Elektrokardiogramm-Signalen (EKG) während MagnetresonanzUntersuchungen, welche zur Triggerung der Messungen und eventuell als Patientenmonitoring dienen, wurde ein EKG-System mit optischer Signalübermittlung und optischer Leistungsversorgung entwickelt.
\end{abstract}

\section{EINLEITUNG}

Eine grosse und tendenziell steigende Anzahl an MR-Sequenzen basiert auf einer Synchronisation der Akquisition mit dem EKG. Das EKG-Signal, das während einer MR-Untersuchung aufgenommen wird, ist allerdings häufig durch das stationäre und durch hochfrequente (RF) Magnetfelder und durch schnell geschaltete Gradientenfelder stark gestört. All diese Effekte machen eine zuverlässige Detektion der R-Zacke im EKG oft sehr schwierig. Um gradienteninduzierte Verzerrungen zu minimieren, müssen die Elektroden einerseits nahe dem Isozentrum des Magneten plaziert werden und andererseits muss die Fläche, welche zwischen Elektroden und den Ableitungsdrähten aufgespannt wird, möglichst klein sein.

Aus diesen Anforderungen folgt, dass ein EKGVorverstärker möglichst nahe bei den EKG-Elektroden plaziert werden sollte. Da der elektrische Schaltkreis für den Vorverstärker aber eine Stromversorgung benötigt, werden häufig dafür Batterien eingesetzt. Batterien enthalten leider aber zumeist ferromagnetische Materialen, welche grosse Bildartefakte verursachen, und können deshalb nicht nahe der Bildebene plaziert werden. Eine vorgängig publizierte Lösung $[1,2]$ beinhaltete eine optische Uebertragung des EKG-Signals vom Vorverstärker beim Patienten aus dem Magneten heraus, hatte aber noch eine Batterie beim EKG-Vorverstärker.

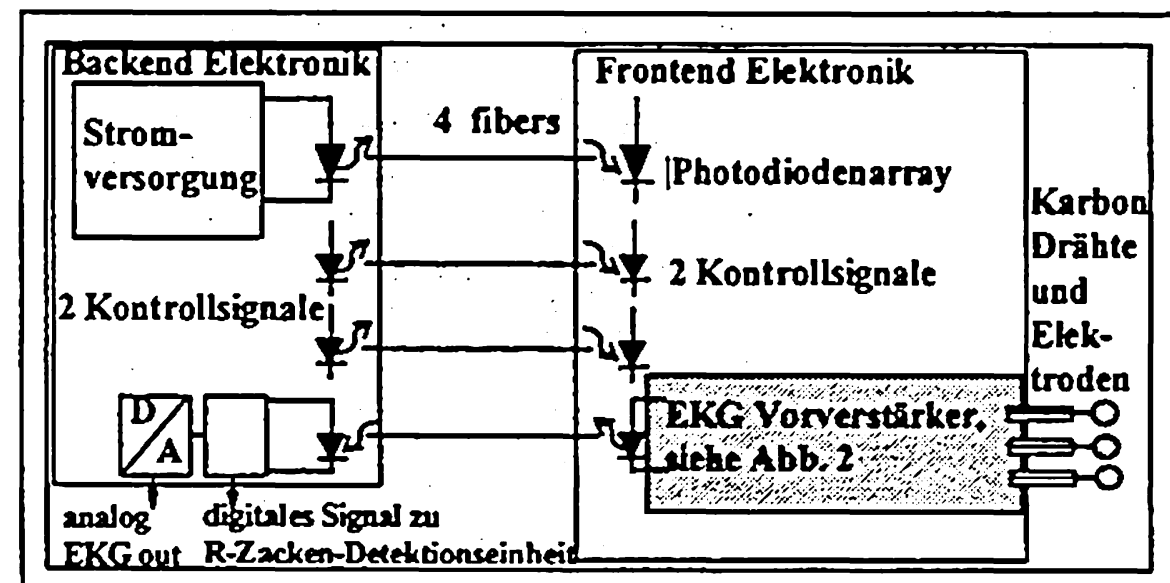

Abbildung 1: Blockdiagramm des Gesamtsystems mit Frontend- und Backend-Einheit und der optischen Uebertragung sämtlicher Signale.
Deshalb wurde ein batterieloses System entworfen, um die freie Kabellänge zwischen Hautelektroden und elektrischem Schaltkreis im Vorverstärker zu minimieren. Der EKG-Vorverstärker ist nahe oder sogar in der Abbildungsebene, nahe den Elektroden plaziert. Er basiert auf einer optischen Leistungsversorgung, optisch übermittelten Kontrollsignalen und einer optischen Uebermittlung des verstärkten und digitalisierten EKG-Signals mittels Glasfasern. Diese Lösung benötigt also keinerlei elektrische Verbindung und auch keine Batterie.

\section{MATERIALIEN UND METHODEN}

Optische Leistungsversorgung (siehe Abb. 1): Eine Laserdiode (SFH 487406, Siemens) erzeugt eine optische Ausgangsleistung von $30 \mathrm{~mW}$ und speist diese in eine 12 Meter lange multi-mode Glasfiber mit einem Kerndurchmesser von 200 Mikrometern.

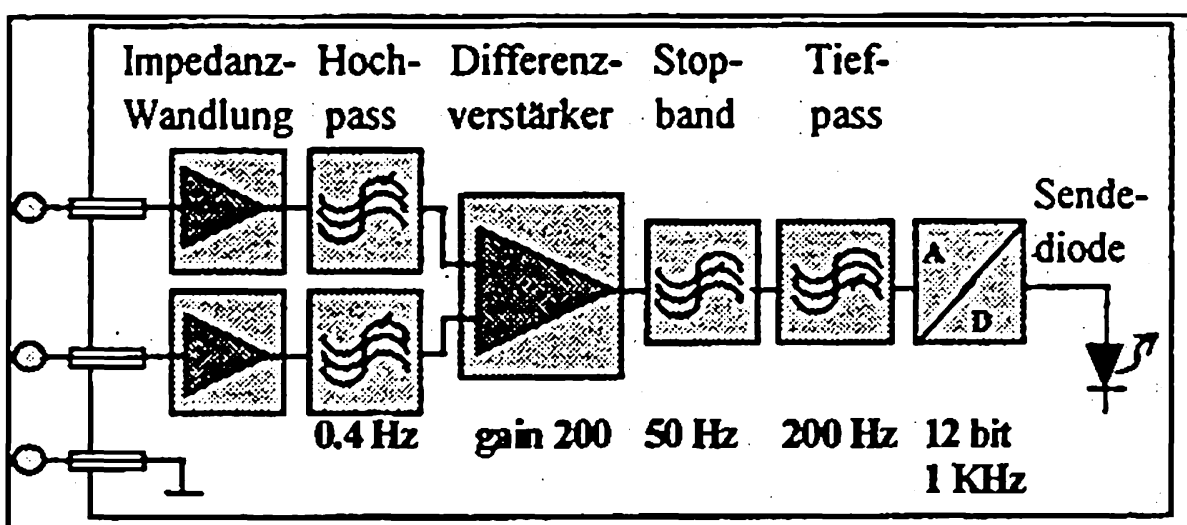

Abbildung 2: Blockschema des EKG-Vorstärkers mit Analog-Digitalwandlung und optischer Signalübertragung.

Mit einem Photodiodenarray (SFH 2115, Siemens) wird empfängerseitig eine elektrische Leistung von ca. $3.5 \mathrm{~mW}$ erhalten für die Speisung des Vorverstärkers.

EKG-Elektroden und -Kabel: Kurze Karbonkabel (mittlere Länge ca $5 \mathrm{~cm}$ ) und metallfreie Karbonelektroden (Carbocone M45, Lynn Medical Systems, USA) wurden benützt.

Frontend-Elektronik (der Teil des Systems im Magneten, wie z.B. EKG-Vorverstärker, optische Konverter, siehe Abbildungen 1 und 2): Mit extrem leistungsarmen Komponenten hat die gesamte Frontend-Elektronik einen Leistungsbedarf von $2.8 \mathrm{~mW}$. Der Prototyp ist mit miniaturisierten SMD (surface mounted devices) aufgebaut und in eine RF-abgeschirmte Box von einer Kantenlänge von $1 \times 3 \times 9 \mathrm{~cm}$ und einem Gewicht von weniger als 50 Gramm eingeschlossen.

Die Signale von den Elektroden passieren zuerst einen Impedanzwandler und werden anschliessend hochpassgefiltert und gelangen zum Differenzverstärker. Darauf folgen ein Stopband-Filter mit der Mittenfrequenz von 50 Hertz und ein Tiefpassfilter. Die 12-bit breite AnalogDigitalwandlung erfolgt mit einer Abtastrate von $1 \mathrm{kHz}$ 
mit der "Successive approximation conversion" Technik (LTC1286, Linear Technology).

Das digitalisierte EKG-Signal wird anschliessend optisch über die Glasfaser übermittelt. Dazu dient eine extrem leistungsarme LED (HLMP-K155, Hewlett Packard) mit einem Leistungsbedarf von lediglich $100 \mathrm{uW}$. Diese tiefe Leistung ist möglich durch die Benützung einer Glasfaser mit einem Kerndurchmesser von $0.6 \mathrm{~mm}$.

Die Backend-Elektronik (siehe Abb, 1) ist als PC-Einschubkarte aufgebaut. Eine Funktion des Backends ist die Generierung von Kontrollsignalen für den digitalen Teil der Frontend-Elektronik und die anschliessende Uebermittlung dieser über Glasfiber (Chip select, Clock).

Das Konzept mit externen Kontrollsignalen hat die folgenden Vorteile: Es wird kein hochfrequenter Quartzkristall als Frequenzreferenz benötigt, welcher zusätzlich beim Frontend Störungen bei der Messung der MR-Signale verursachen könnte. Zusätzlich ist damit das System expandierbar; mehrere Frontend-Einheiten können völlig synchron miteinander benützt werden, womit das EKGSignal synchron genau zu gleichen Zeitpunkten in mehreren Kanälen abgetastet werden kann.

Das optisch übermittelte EKG-Signal wird im Backend in ein elektrisches Signal zurückgewandelt und wird als digitales serielles Signal zur R-Zacken-Detektion und EKGTriggerung dem Scanner zugefuhrt. Das EKG-Signal wird überdies wieder in ein analoges Signal gewandelt, damit es auf einem Oszilloskop jederzeit überwacht werden kann.

\section{ERGEBNISSE}

Ein Prototyp wurde mit SMD-Bausteinen aufgebaut. Tests wurden mit Probanden auf einem Philips Gyroscan ACS/NT Ganzkörper-MR-Gerät mit einer Feldstärke von 1.5 Tesla durchgefuhrt.

Die Abbildung 3 zeigt das abgeleitete EKG-Signal eines freiwilligen Probanden während dem Zeitintervall von 4 aufeinanderfolgenden Herzschlägen. Die obere Linie repräsentiert das EKG, so wie es während einer MR-Untersuchung aus dem Magneten heraus gewonnen wurde. Die untere Linie in Abbildung 3 ist zur besseren Lesbarkeit leicht links verschoben worden. Sie repräsentiert das EKG-Signal, gewonnen an der gleichen Person, unmittelbar anschliessend an die MR-Untersuchung, jedoch akquiriert ausserhalb des Magneten, so wie es mit der präsentierten Hardware aufgenommen wurde.

\section{DISKUSSION}

Das Konzept und der Prototyp mit der batterielosen EKGElektronik in einer abgeschirmten miniaturisierten Box nahe den Elektroden minimiert die Länge der Karbondrähte an den Elektroden. Die Abtastrate von $1 \mathrm{kHz}$ mit einer Dynamik von 12 bit ermöglichen ein hochqualitatives EKG-Signal. Da die Kontrollsignale extern generiert und über Glasfiber zur Frontend-Elektronik abermittelt werden, ist die Frontend-Einheit echt kaskadierbar. Das heisst, mehrere der vorgestellten EKG-VerstärkerEinheiten mit je 3 Elektroden können simultan betrieben werden. Mit mehr als 3 EKG-Elektroden wird es möglich, nicht nur eine Projektion, sondem eine dreidimensionale Vektor-Repräsentation des EKG-Signals aus den einzelnen Signalen abzuleiten. Störungen und Verzerrungen des EKG-Signals, beispielsweise durch schnell geschaltete Gradientenfelder folgen aber nicht dem rotierenden EKGVektor. Deshalb besteht die Hoffnung, dass diese Störungen mit geeigneten Algorithmen aus einer Vektor-
Darstellung im EKG-Signal eliminiert oder mindestens minimiert werden könnten.

Eine Prozedur mit multiplen EKG-Elektroden hilft auch, die R-Zacke in Patienten mit abnormaler elektrischer Herzachse oder mit Erregungsausbreitungsstörungen zuverlässiger detektieren zu können. Mit einem modellbasierten Ansatz könnte der Einfluss des statischen Magnetfeldes auf das Elektrokardiogramm reduziert werden. Als Demonstration dieses Effektes betrachte man die Abb. 3, obere Linie: Die R-Zacken sind praktisch identisch zu denjenigen, welche ausserhalb des Magneten

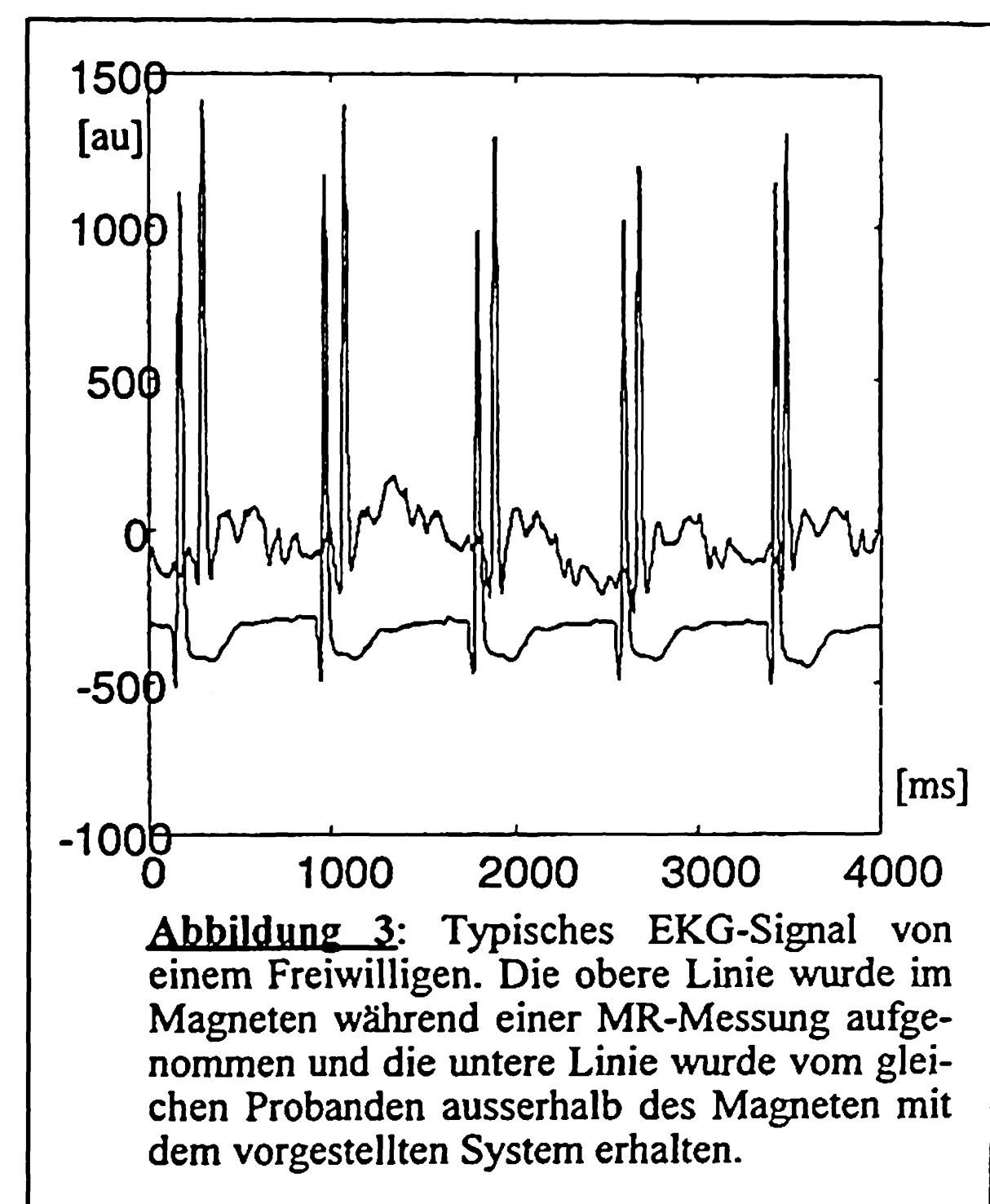

gewonnen wurden. Das EKG-Signal zwischen den aufeinanderfolgenden QRS-Komplexen variiert aber stärker, vor allem ist dieser Effekt im Intervall S-T sehr stark ausgeprägt.

Ausgedehntere Untersuchungen an Patienten mit abnormem EKG sind noch notwendig und noch durchzufuihren. Die Möglichkeit einer Kaskadienung der Akquisition eines zuverlässigen und qualitativ guten EKG-Signals liefert aber nicht nur eine zuverlässigere R-ZackenDetektion zur Triggerung der Messung. Darüber hinaus könnte es auch zum Teil von diagnostischem Wert werden. Dies wäre bei der MR-Untersuchung von unstabilen Herzpatienten oder während Stress-Untersuchungen im MR-scanner von vitaler Bedeutung.

\section{LITERATUB}

[1] J. Felblinger, C. Lehmann, C. Boesch: Magn. Reson. Med., 32:523-529 (1994)

[2] J. Felblinger, et.al. Radiology, 197:311-313 (1995) 